\title{
Die Funktion der Antennen, Palpen und Tarsen von Aedes aegypti L. beim Aufsuchen des Wirtes
}

von

\section{U. RAHM}

Basel

Mit 3 Textabbildungen und 4 Tabellen.

Bei unseren Attraktionsversuchen mit Aedes aegypti an Menschen stellten wir seinerzeit fest, dass eine menschliche Hand im Wahlversuch viel mehr Aedes-Weibchen anlockt, als eine „künstliche Hand“, die aus einer $35^{\circ} \mathrm{C}$ warmen, mit einem feuchten Lappen bedeckten Flasche bestand. Wahlversuche mit einem besonderen Dispositiv, mit welchem die vom Menschen abgegebenen „Duftstoffe“ getestet werden konnten, haben gezeigt, dass der Duft für die Attraktion der Stechmücken ausschlaggebend ist. Diese Duftstoffe, kombiniert mit Wärme und Feuchtigkeit, locken ebensoviel Aedes-Weibchen an wie eine Hand; Wärme und Feuchtigkeit allein attrahieren hingegen wesentlich weniger Mücken (RАнм 1956, $1957 a, 1957 b$, 1958). Im Zusammenhang mit diesen Ergebnissen stellten wir uns die Frage, welche Sinnesorgane die Weibchen der Gelbfiebermücke befähigen, die Duftstoffe einerseits und Wärme und Feuchtigkeit andererseits wahrzunehmen und zu unterscheiden. Rотн und Willis (1952) untersuchten die Reaktion von Aedes bei verschiedener Luftfeuchtigkeit und fanden durch sukzessive Amputation der Antennenglieder, dass auf diesen anscheinend Hygrorezeptoren vorhanden sind. Rотн (1951) führte an Aedes und Anopheles eine Reihe vom Amputationsversuchen durch und kam zum Schluss, dass die Aedes-Weibchen hauptsächlich mit 
ihren Antennen und Palpen den Wirt ausfindig machen und dass diese beiden Organe auch die Stimuli empfangen, die den Stechreflex auslösen. Tarsen und Stechwerkzeuge spielen bei der Attraktion keine Rolle. Rотн schreibt in seiner Diskussion: „The results with Aedes aegypti using temperature alone as the stimulus, parallel closely those obtained using the arm and hand to attract females and induced probing. Since in using the arm as a stimulus one is dealing not only with chemical but thermal stimuli as well, it is not possible to conclude that the antennae function as distance chemoreceptors, although this is highly probable." Wir versuchten mit unserer Testmethode die Frage näher abzuklären. Anhand von Wahlversuchen zwischen einer menschlichen Hand (Duft, Wärme, Feuchtigkeit) und einem „,künstlichen Arm“(Wärme, Feuchtigkeit), kombiniert mit verschiedenartigen Amputationen, sollte ermittelt werden, ob die Aedes-Weibchen in der Lage sind, Duftstoffe wahrzunehmen.

\section{Material und Methode}

Zur Amputation der Körperanhänge entnahmen wir AedesWeibchen einem Zuchtkäfig und brachten sie jeweils in Gruppen von 5-10 Stück in ein eisgekühltes Reagensglas, um sie durch Kälte zu immobilisieren. Die darauffolgende Operation wurde meist mit einer feinen Uhrmacherpinzette ausgeführt, gelegentlich auch mit einer Augenschere. Die Kontrolltiere aus dem gleichen Zuchtkäfig sezten wir derselben Kälteeinwirkung aus. Die Mücken erholten sich innerhalb einer Stunde, so dass, wenn am Vormittag amputiert wurde, am Nachmittag die Versuche folgen konnten. Die Mücken liessen wir während 48 Stunden vor Versuchsbeginn hungern (RАнм 1956) und führten an einem Tage mit denselben Tieren nicht mehr als 4-5 Versuche durch. Als Versuchskäfige verwendeten wir zwei Typen: 1. Ein Käfigtyp, der bereits in unseren früheren Wahlversuchen verwendet wurde und in der Publikation Rанм 1956 eingehend beschrieben ist (Wahlkäfig, Grösse: 45 / 45 / $50 \mathrm{~cm}$ ). Ein solcher Wahlkäfig enthielt jeweils 50 amputierte Weibchen, in einem zweiten genau gleichen Käfig, befanden sich 50 Kontrolltiere. 2. Ein kleinerer Käfigtyp (Grösse: 25 / 25 / $30 \mathrm{~cm}$ ) enthielt jeweils 30 amputierte Tiere und ein zweiter identischer beherbergte 30 Kontrolltiere (= Kleinkäfig). Im Gegensatz zum 
Wahlkäfig wurde somit in diesem Kleinkäfig nur ein Objekt ausgetestet. Die Testdauer betrug 10 Minuten, wobei die attrahierten Aedes-Weibchen alle Minuten gezählt wurden (vergl. Raнм 1956).

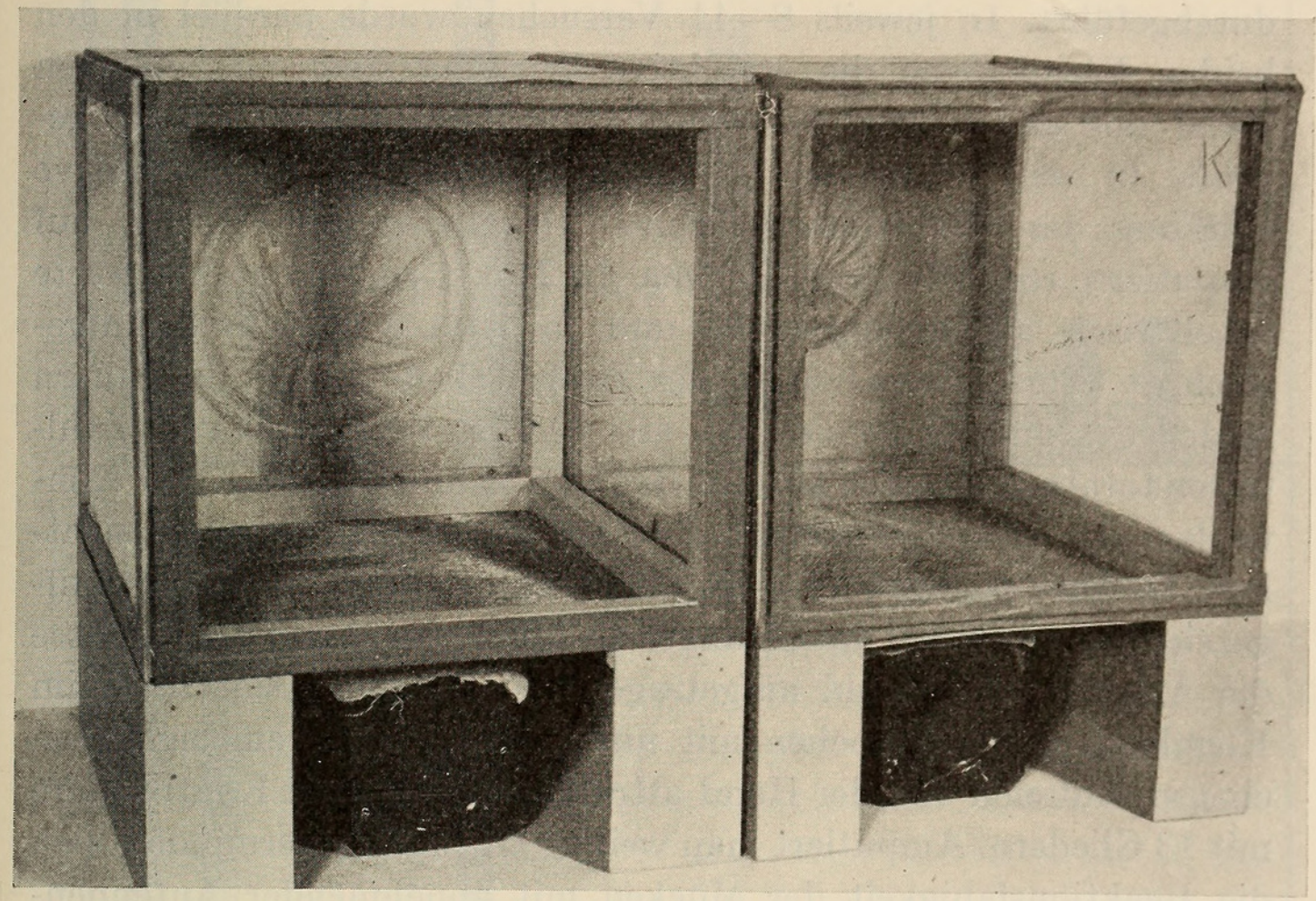

Ав в. 1.

Testen im Kleinkäfig. Abgebildet ist der Test mit der feucht/warmen Flasche, links amputierte Mücken, rechts Kontrollen.

\section{VERSUCHE}

a) Antennenamputationen: Die Versuche von Rотн (1951) weisen schon darauf hin, dass auf den Antennen diejenigen Sinnesorgane vorhanden sein müssen, welche bei der Fernattraktion die Hauptrolle spielen. Auch Untersuchungen an anderen Insekten haben ergeben, dass die Antennen wichtige Sinnesorgane tragen (Marshall, Wigglesworth, Dethier, Wiesinger, Kraus). Wie bereits angedeutet, versuchten wir zu erfahren, ob auf den Antennen neben Thermo- und Hygrorezeptoren auch Chemorezeptoren zur Wahrnehmung der Duftstoffe vorhanden seien. In verschiedenen Versuchsserien wurden amputiert: das letzte, d. h. 13. Antennenglied, sodann die letzten 4, 7, 10,12 und alle 13 Glieder. Die Total- 
amputation der Antennen wird erschwert durch den Umstand, dass das 1. Glied mit seiner Basis in der Grube des Pedicellus sitzt, so dass dessen letzter Rest meist nicht entfernt werden kann.

Eine erste Versuchsserie wurde mit dem Kleinkäfig (Abb. 1) durchgeführt. In jeweils 8-11 Versuchen wurde parallel in den beiden Kleinkäfigen die attraktive Wirkung einer menschlichen Hand auf amputierte Aedes-Weibchen und auf Kontrolltiere geprüft. In weiteren 8-10 Tests untersuchten wir die attraktive Wirkung des „künstlichen Armes“ (feucht/warme Flasche) auf amputierte resp. Kontroll-Mücken. In Tab. 1 sind die Zahlenwerte tabellarisch zusammengestellt und in Abb. 2 graphisch aufgezeichnet (Einzelversuche). In der Zahlentabelle stellen die Ziffern in den einzelnen Kolonnen die pro 10-Min.-Versuch bei 10 Zählungen total attrahierten Mücken dar $(\mathrm{Am}=$ amputierte, $\mathrm{Ko}=\mathrm{Kon}$ trollen). Der Durchschnittswert ist aus der untersten Zeile ersichtlich. In Abb. 2 oben sind diese Durchschnittswerte als Säulen aufgezeichnet, wobei auf der Ordinate die Mückenzahl, auf der Abszisse die Antennengliederzahl aufgetragen ist. Es ergibt sich, dass im Kleinkäfig Aedes-Weibchen mit nur 12 resp. 9 Antennengliedern ebenso zahlreich von der Hand attrahiert werden wie Kontrolltiere mit 13 Gliedern. Amputiert man weitere Glieder, so vermindert sich die Reaktionsfähigkeit der Mücken mit abnehmender Antennengliederzahl. Bietet man den gleichen Versuchstieren eine feucht/ warme Flasche dar, so reagieren die Tiere mit nur 3 Gliedern praktisch noch gleich gut auf diese Reizquelle wie mit 12, resp. 9 oder 6. Lässt man den Aedes-Weibchen nur noch 2 resp. 1 oder 0 Glieder, so nimmt die Zahl der attrahierten Tiere merklich ab. Mücken mit amputierten Antennengliedern reagieren also verschieden, je nachdem ihnen eine menschliche Hand oder eine feucht/ warme Flasche als Reizquelle dargeboten wird.

In einer zweiten Versuchsserie, bei welcher in den zwei grösseren sog. Wahlversuch-Käfigen die Reaktion von amputierten AedesWeibchen und Kontrolltieren untersucht wurde, hatten die Tiere die Wahl zwischen einer menschlichen Hand und einer feucht/ warmen Flache. In Tab. 2 sind die Zahlenwerte eingetragen und in Abb. 2 unten sind diese graphisch dargestellt. Hier ergibt sich, dass die Mückenweibchen, wenn 6 und mehr Glieder vorhanden sind, die menschliche Hand der Flasche vorziehen, dass hingegen Tiere mit nur $0-3$ Gliedern vorzugsweise die Flasche anfliegen. 


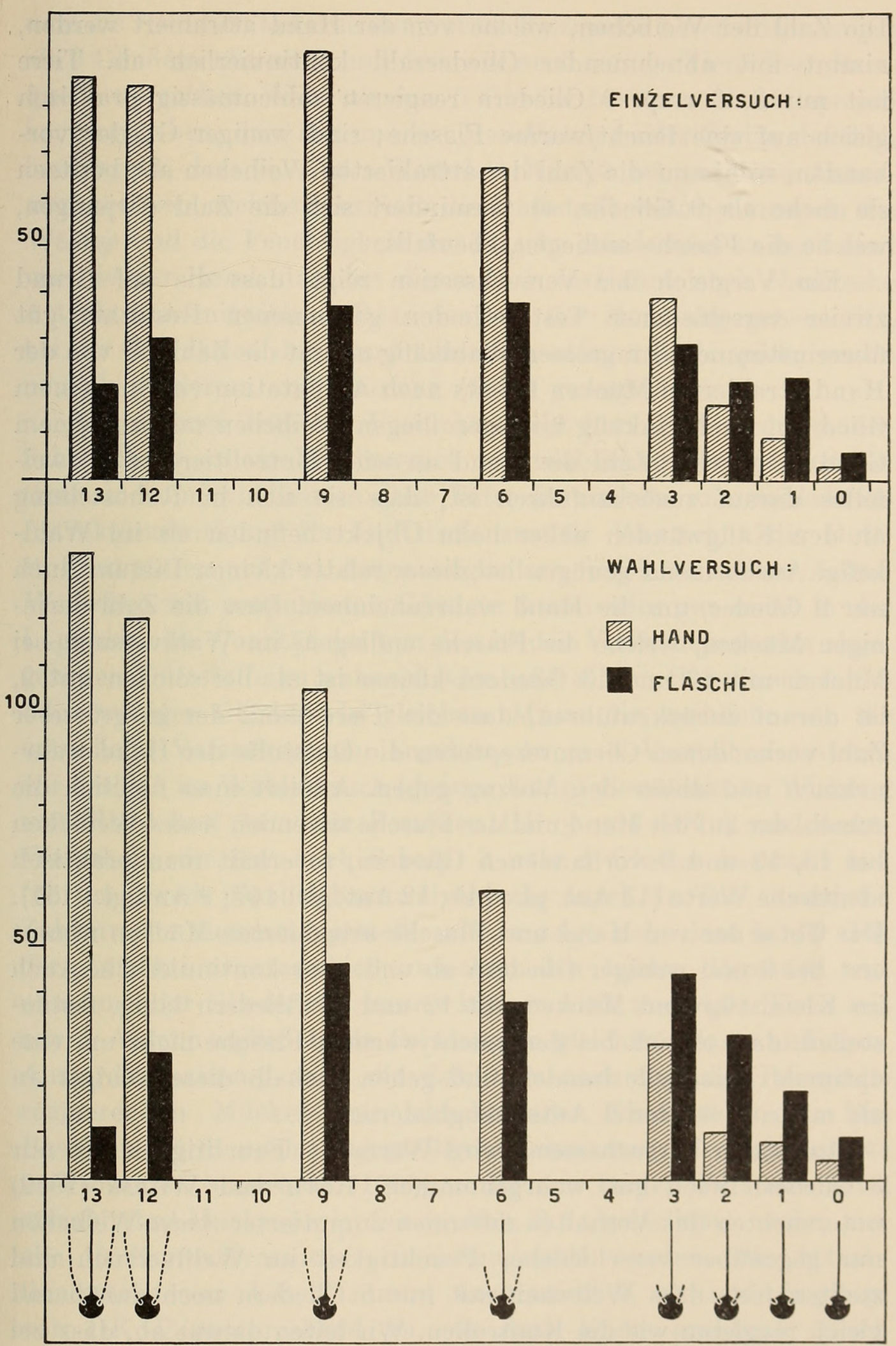

Aвв. 2.

Graphische Darstellung der Resultate von Tab. 1 und 2. Oben: Kleinkäfigversuche (Einzelversuche.) Unten: grosser Käfig für Wahlversuche.

Die Erklärung der Säulen-Muster gilt für beide Darstellungen. 
Die Zahl der Weibchen, welche von der Hand attrahiert werden, nimmt mit abnehmender Gliederzahl kontinuierlich ab. Tiere mit nur 3, 6 resp. 9 Gliedern reagieren zahlenmässig praktisch gleich auf eine feucht/warme Flasche; sind weniger Glieder vorhanden, so nimmt die Zahl der attrahierten Weibchen ab; besitzen sie mehr als 9 Glieder, so vermindert sich die Zahl derjenigen, welche die Flasche anfliegen, ebenfalls.

Ein Vergleich der Versuchsserien zeigt, dass die auf Grund zweier verschiedener Testmethoden gewonnenen Resultate gut übereinstimmen. Im grossen Wahlkäfig nimmt die Zahl der von der Hand atrahierten Mücken bereits nach Amputation von nur einem Glied ab, im Kleinkäfig hingegen fliegen Weibchen mit nur einem Glied in gleicher Zahl die Hand an wie Kontrolltiere, was zweifellos darauf zurückzuführen ist, dass sie sich in Ruhestellung an den Käfigwänden näher beim Objekt befinden als im Wahlkäfig. Anscheinend genügen bei dieser relativ kleinen Distanz auch nur 9 Glieder, um die Hand wahrzunehmen. Dass die Zahl derjenigen Mücken, welche die Flasche anfliegen, im Wahlversuch bei Mücken mit 12 und 13 Gliedern kleiner ist als bei solchen mit 9, ist darauf zurückzuführen, dass die Tiere dank der in grösserer Zahl vorhandenen Chemorezeptoren die Dufstoffe der Hand wahrnehmen und dieser den Vorzug geben. Addiert man nämlich die Anzahl der auf der Hand und der Flasche sitzenden Aedes-Weibchen bei 13, 12 und 9 vorhandenen Gliedern, so erhält man praktisch identische Werte (13 Ant. gl. : 145; 12 Ant. gl. : 147; 9 Ant. gl. : 151). Das Total der von Hand und Flasche attrahierten Mücken nimmt erst bei 6 und weniger Gliedern ab und zwar kontinuierlich. Auch im Kleinhäfig sind Mücken mit 12 und 13 Gliedern fähig, festzustellen, dass es sich bei der feucht/warmen Flasche nicht um eine optimale Reizquelle handelt und gehen deshalb diese weniger an als mit nur 9 oder 6 Antennengliedern.

In beiden Versuchsserien wird Wärme + Feuchtigkeit mit nur 3 Gliedern noch gut wahrgenommen. Rотн und Willis (1952) untersuchten das Verhalten antennen-amputierter Aedes-Weibchen nur gegenüber verschiedener Feuchtigkeit im Wahlversuch und stellten fest, dass Weibchen mit nur 5 Gliedern noch annähernd gleich reagieren wie die Kontrollen. Wir leiten daraus ab, dass bei unserer feucht/warmen Flasche die Aedes-Weibchen vor allem auf deren Feuchtigkeit reagieren. Versuche von Rотн (1951) zeigen, 
dass die Reaktion auf eine nur warme Reizquelle bereits bei weniger als 9 Gliedern abnimmt und dass nur noch wenige Tiere diese Reizquelle aufsuchen, wenn sie nur 5 Glieder besitzen. Es sei speziell hervorgehoben, dass aus unseren Resultaten beider Serien deutlich hervorgeht, dass die Aedes-Weibchen Rezeptoren auf ihren Antennen besitzen, welche spezifisch die Duftstoffe wahrnehmen, da die Wärme und die Feuchtigkeit der Flasche mindestens so hoch sind wie die der Hand, dass bei dieser aber zusätzlich noch chemische Attraktoren mitwirken (Dass das von der Haut abgegebene $\mathrm{CO}_{2}$ bei der Attraktion der Hand keine wesentliche Rolle spielt, wurde neulich wiederum von Brower (1958) gezeigt).

Aus unseren früheren Versuchen geht hervor, dass die männlichen Personen im Wahlversuch mit weiblichen Personen attraktiver sind und wir führten diese Tatsache auf eine unterschiedliche Attraktionswirkung der Duftstoffe zurück. Wenn nun die Duftstoffrezeptoren auf den Antennengliedern liegen, so sollten AedesWeibchen mit amputierten Gliedern kaum oder gar nicht in der Lage sein, die Qualität der von den beiden Versuchspersonen abgegebenen Duftstoffe zu unterscheiden und sollten theoretisch mit 3 oder weniger Gliedern in gleichem Masse die weibliche wie die männliche Versuchsperson angehen. In je 5 Versuchen testeten wir deshalb im Wahlversuch (grosser Käfig) die attraktive Wirkung: von Hand und Arm der Personen UR $\hat{\sigma}$ und DK $q$ mit AedesWeibchen, die nur noch 6 resp. 3 oder 1 Glieder besassen und führten jeweils parallel Kontrollversuche durch. In Tab. 3 sind die Zahlenwerte zusammengestellt und es zeigt sich, dass in der Tat der Attraktionsunterschied zwischen den beiden Personen mit abnehmender Gliederzahl immer kleiner wird und dass Tiere mit nur 1 Antennenglied beide Personen in gleichem Masse anfliegen. Ausserdem nimmt auch die Zahl der noch auf die Reizquelle reagierenden Mücken mit abnehmender Gliederzahl ab. Das Resultat wird noch augenfälliger, wenn man die Zahlenwerte in Prozenten ausdrückt $(100 \%=$ die von beiden Personen total attrahierten Mücken) wie dies in Abb. 3 dargestellt ist, wobei hier alle 15 Kontrollversuche zusammengefasst wurden. Die Attraktionsstärke von UR $\hat{o}$ nimmt kontinuierlich ab und diejenige von $\mathrm{DK}$ \& entsprechend $\mathrm{zu}$, so dass beide praktisch gleich attraktiv sind, wenn nur ein Antennenglied bei den Mücken vorhanden ist. 


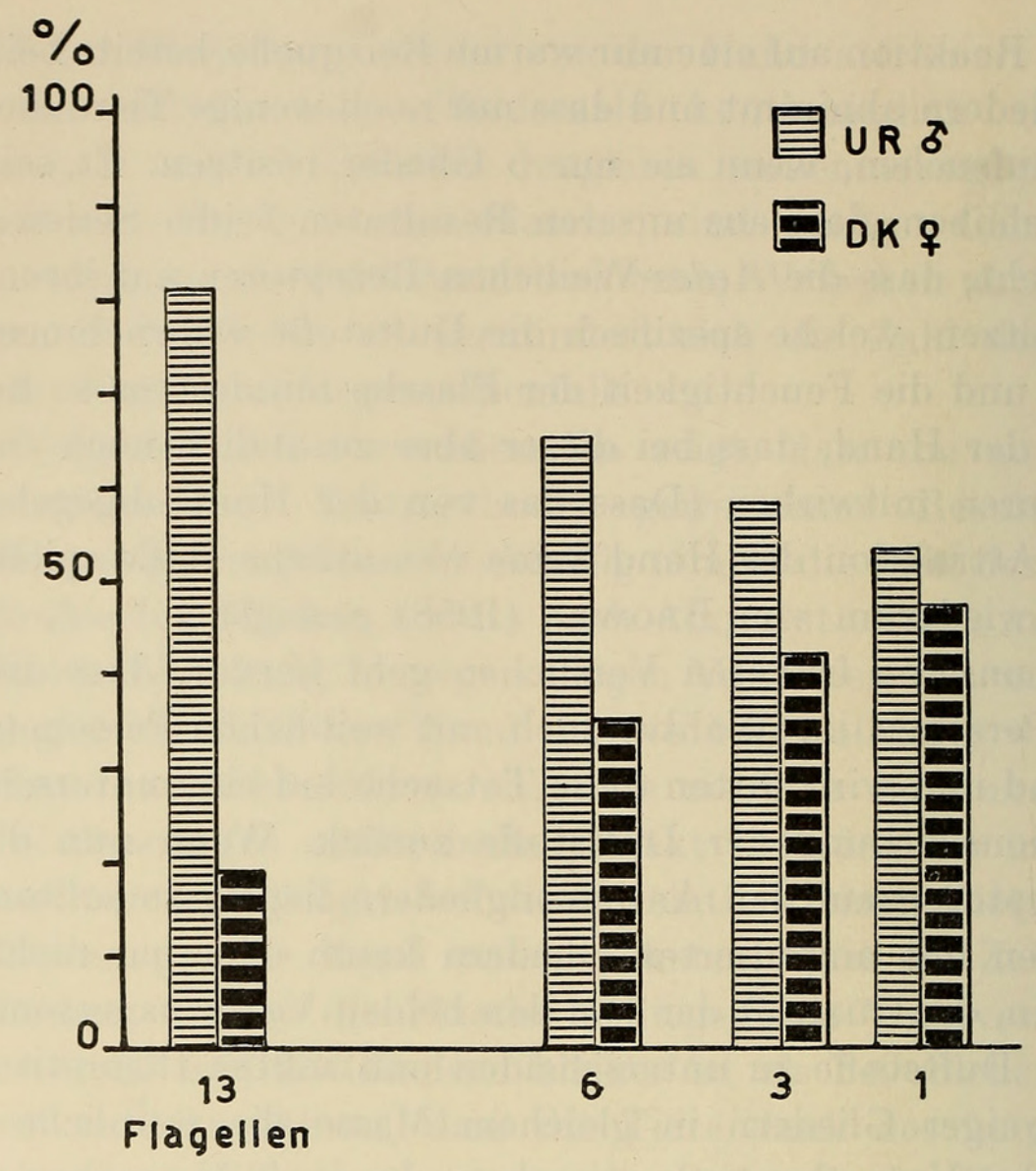

Авв. 3.

Attraktivität von UR ô und D K 9 im Wahlversuch gegenüber Aedes-Weibchen mit verschiedener Antenengliederzahl (13=Kontrolltiere mit 13 Gliədern)

b) Amputation der Palpen und Tarsen. Als Ergänzung der Antennenamputationen wurden auch einige Tarsen- und Palpenamputationen durchgeführt. Rотн (1951) hat nämlich bei Anopheles quadrimaculatus festgestellt, dass auch nach kombinierter Antennen- und Palpen-Totalamputation noch ziemlich viele Tiere den Wirt anfliegen und saugen, aber nur wenn die Mücken sich in der Nähe des Wirtes befinden. Bei Aedes aegypti war dies nach derselben Operation nicht der Fall. Rотн vermutet, dass bei Anopheles auch die Tarsen des 3. Beinpaares Träger von Sinnesorganen sind. Bekanntlich führen auf einer Unterlage sitzende Stechmückenweibchen mit dem 3. Beinpaar „Ruderbewegungen“ aus. Um zu erfahren, ob auch bei unserer Testmethode die Tarsenglieder von Aedes bei der Fernorientierung vielleicht mit eine Rolle spielen, amputierten wir die Tarsenglieder des 3. Beinpaares bei jeweils 30 Aedes-Weibchen und führten im Kleinkäfig 13 Tests aus, wobei 
gleichzeitig mit 30 Tieren Kontrollversuche im zweiten Käfig ausgeführt wurden. Die Ergebnisse sind in Tab. 4 in Kolonne I zusammengefasst und sie zeigen eindeutig, dass durchschnittlich fast gleichviel amputierte wie Kontroll-Mücken die Hand anfliegen.

Werden nun bei intakten Antennen die Palpen beidseitig amputiert, so sind, wie unsere Kleinkäfigversuche zeigen (Tab. 4 II), die Aedes-Weibchen durchaus in der Lage die Hand zu lokalisieren und den Saugakt zu vollziehen. Bei simultaner Amputation von Antennen und Palpen ist jede Fernotientierung ausgeschaltet; gelangt zufällig eines Aedes auf die Drahtgaze dicht über der Hand, so führt sie jetzt keine Stechbewegungen mehr aus (Tab. 4 III). Die Palpen scheinen somit für die Wirtsfindung bedeutungslos. Immerhin zeigen Versuche von Rотн (1951), dass diese Anhänge offenbar für die Auslösung des Stechaktes von einiger Bedeutung sein können, nämlich (vielleicht kompensatorisch) nach Antennenamputation. Er fand, dass antennen-amputierte Aedes-weibchen noch stechen und saugen, solange sie Palpen besitzen.

\section{ZUSAMMENFASSUNG}

1. Versuche mit einem Käfig für Wahltests und mit einem Kleinkäfig für Einzeltests, zeigten, dass Aedes-Weibchen in der Lage sind, eine menschliche Hand von einer feucht/warmen Flasche zu unterscheiden, und die Hand vorziehen.

2. Antennenamputationen lassen erkennen, dass diese Organe für die Wirtsfindung und die Fernattraktion notwendig sind. Werden einzelne Antennenglieder amputiert, so nimmt mit abnehmender Antennengliederzahl die Anzahl der von der Hand attrahierten Aedes-Weibchen kontinuierlich ab, während eine feucht/ warme Flasche auch noch von vielen Aedes wahrgenommen wird, die nur noch wenige Antennenglieder besitzen (Tab. 1, 2 und Abb. 2).

3. Die Wahlversuche zeigen, dass die Aedesweibchen auf ihren Antennen Rezeptoren für „Duftstoffe“ und solche für Feuchtigkeit und Wärme besitzen. Attraktionsunterschiede zwischen einer männlichen und einer weiblichen Versuchsperson sind von Tieren mit partiell amputierten Antennen nicht mehr feststellbar.

4. Die Tarsen des 3. Beinpaares sowie die Palpen sind für die Fernorientierung nicht von Bedeutung. Antennenlose Tiere können noch stechen und saugen, wenn die Palpen intakt sind. 


\section{LITERATURVERZEICHNIS}

Brouwer, R. 1958. Geurverschillen bij de mens als oorzaak van interindividuele verschillen in attractie voor malariamuskieten. Diss. Leiden. 110 p.

Dethier, V. G. 1957. The Sensory Physiology of Blood-Sucking Arthropods. Exp. Parasitol., 6, 1, pp. 68-122.

Kraus, C. 1957. Versuch einer morphologischen und neurophysiologischen Analyse des Stechaktes von Rhodnius prolixus Stal 1858. Acta Tropica, 14, 1, pp. 36-87.

Marshall, J. 1935. The location of olfactory receptors in insects; a review of experimental evidence. Trans. Roy. Ent. Soc. London, 83, pp. 49-72.

Rанм, U. 1956. Zum Problem der Attraktion von Stechmücken durch den Menschen. Acta Tropica, 13, 4, pp. 319-344.

- 1957 a. Wichtige Faktoren bei der Attraktion von Stechmücken durch den Menschen. Rev. Suisse Zool., 64, pp. 236-246.

- 1957 b. Zur Bedeutung des Duftes und des Schweisses bei der Attraktion von Aëdes aegypti durch den Menschen. Acta Tropica, 14, 3, pp. 208-217.

- 1958. Die attraktive Wirkung der vom Menschen abgegebenen Duftstoffe auf Aëdes aegypti L. Z. Tropenmed. u. Parasit. 9, 2, pp. 146-156.

Roth, L. M. 1948. A Study of Mosquito Behavior. An Experimental Laboratory Study of the Sexual Behavior of Aedes aegypti L. Amer. Midland Naturalist, 40, 2, pp. 265-352.

- 1951. Loci of sensory end-organs used by mosquitoes Aëdes aegypti and Anopheles quadrimaculatus in receiving host stimuli. Ann. Ent. Soc. America, 44, 1, pp. 59-73.

Roth, L. M. and Willis, E. R. 1952. Possible hygroreceptors in Aëdes aegypti and Blatella germanica. J. Morphol., 91, pp. 1-14.

Wiesinger, D. 1956. Die Bedeutung der Umweltfaktoren für den Saugakt von Triatoma infestans. Acta Tropica, 13, 2, pp. 97-141.

Wigglesworth, V. B. 1955. Physiologie der Insekten. Birkhäuser, Basel. 


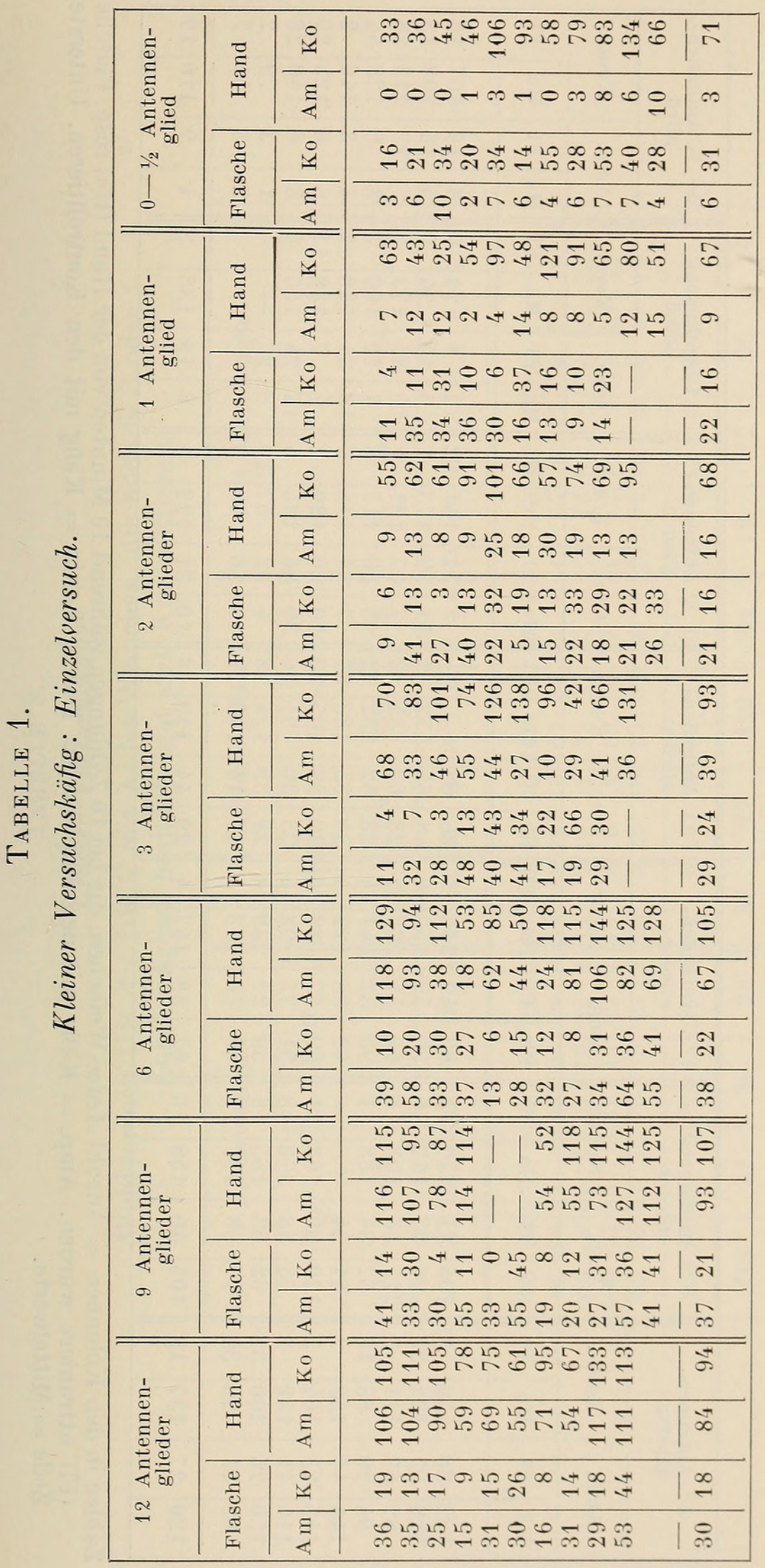




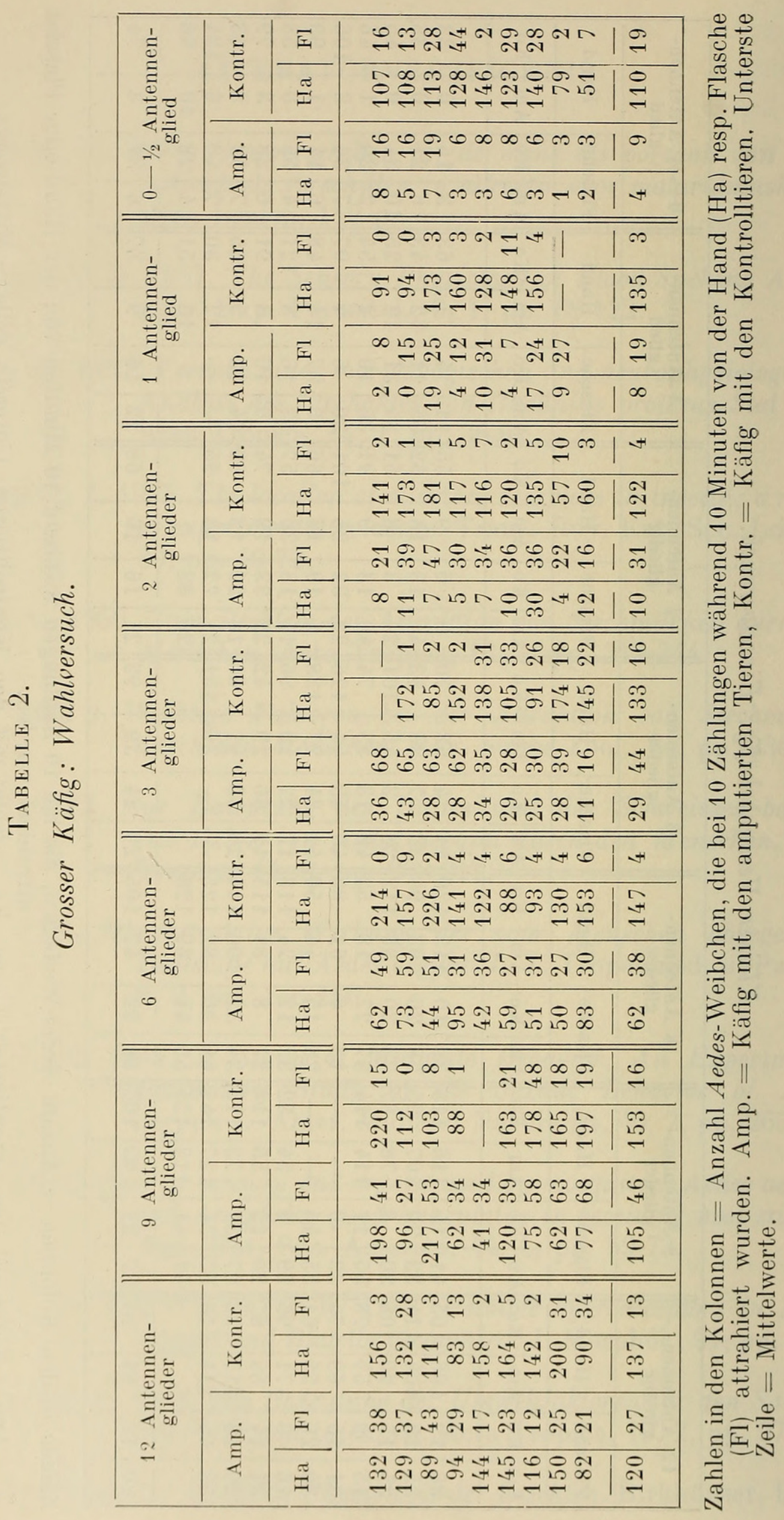




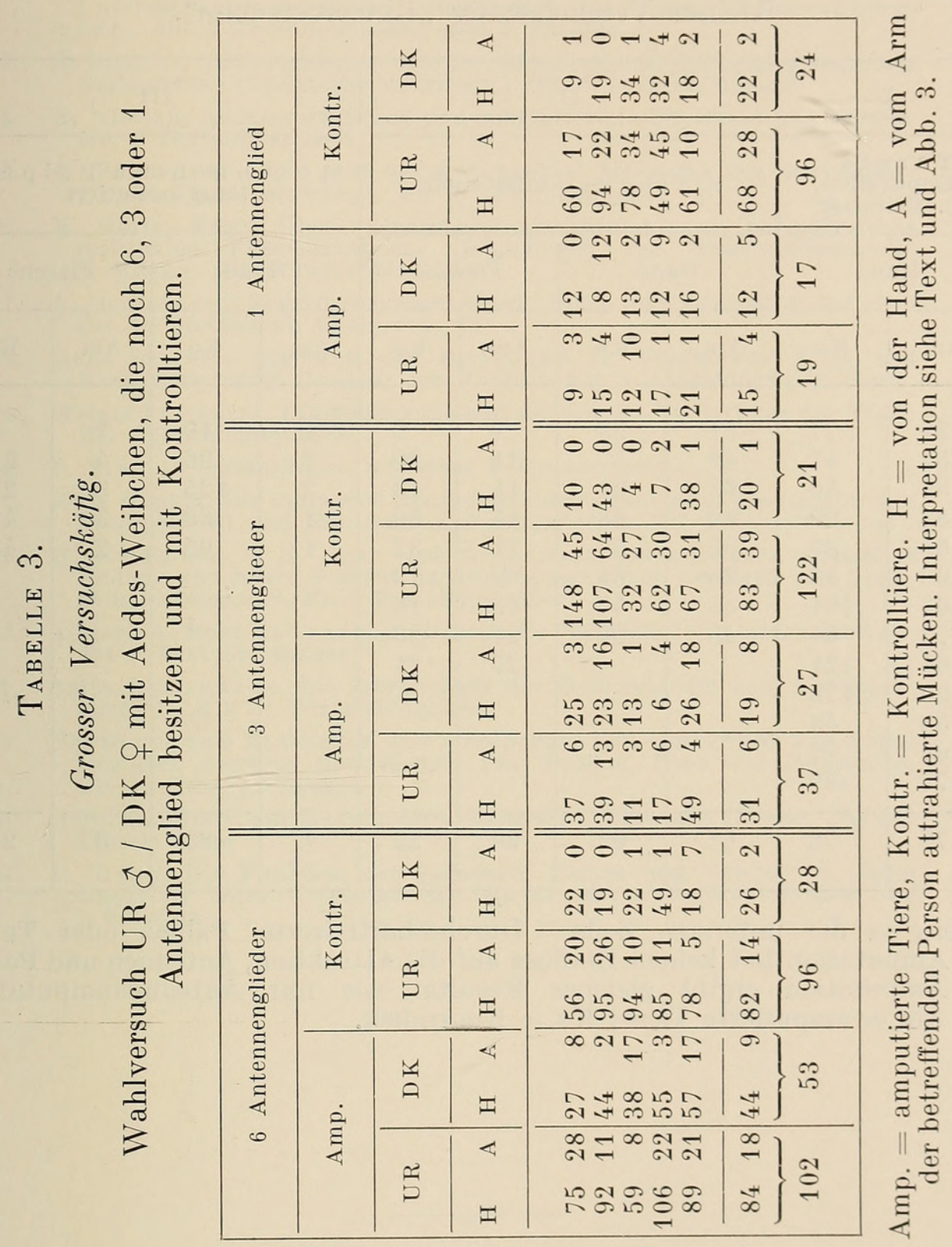


TABELLE 4.

Palpen-, Tarsen- und Antennen/Palpen-Amputation.

Kleiner Versuchskäfig „Einzelversuche“.

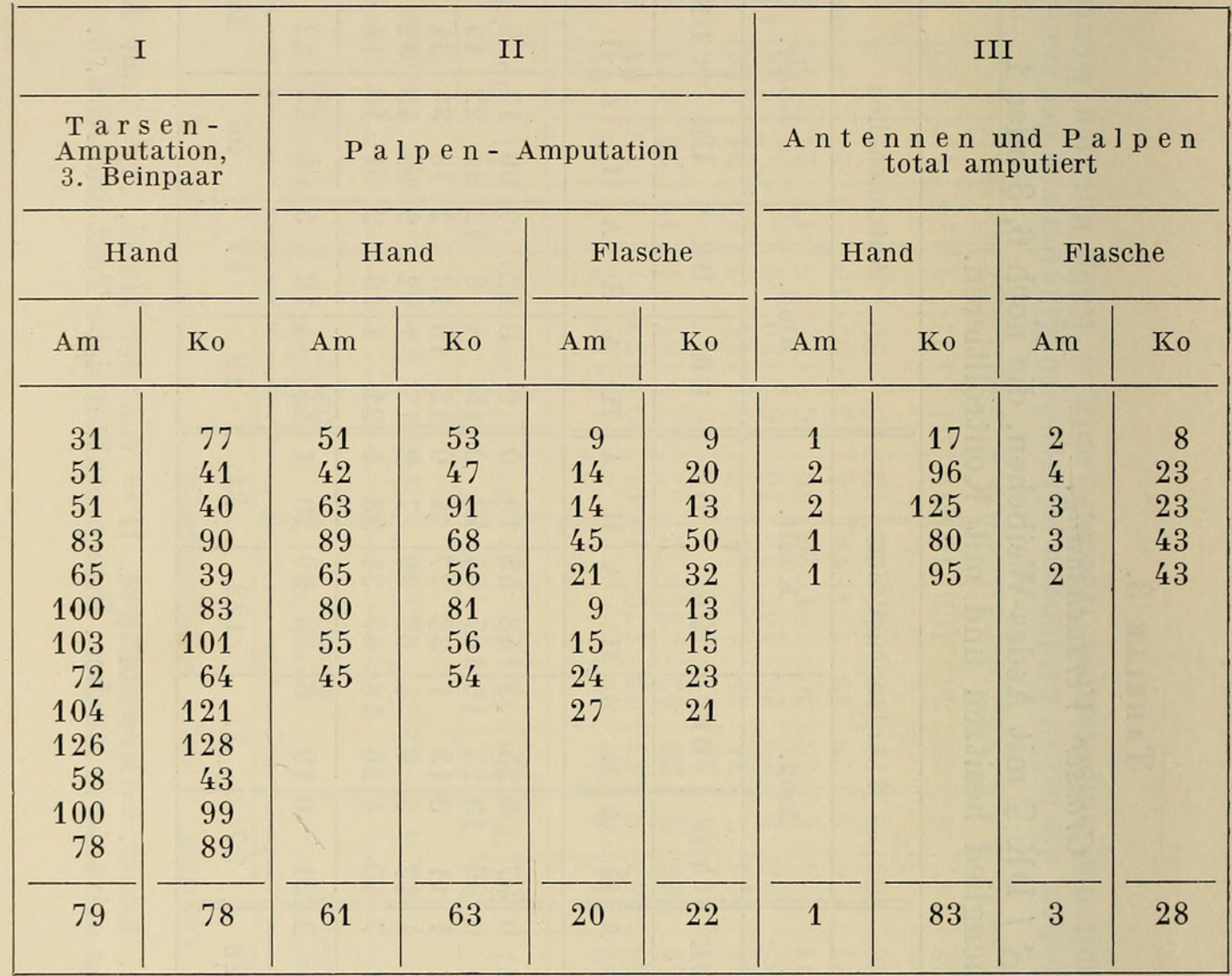

Ziffern in der untersten Zeile $=$ Durchschnittswerte. Palpen oder Tarsen Amputation hat keinen Einfluss auf die Attraktion, Antennen und Palpen Amputation ergibt gleiches Resultat wie nur Antennenamputation. $\mathrm{Am}=$ amputierte Tiere; Ko $=$ Kontrollen. 


\section{$2 \mathrm{BHL}$ Biodiversity Heritage Library}

Rahm, U. 1958. "Die Funktion der Antennen, Palpen und Tarsen von Aedes aegypti L. beim Aufsuchen des Wirtes." Revue suisse de zoologie 65, 779-792. https://doi.org/10.5962/bhl.part.75553.

View This Item Online: https://www.biodiversitylibrary.org/item/126657

DOI: https://doi.org/10.5962/bhl.part.75553

Permalink: https://www.biodiversitylibrary.org/partpdf/75553

\section{Holding Institution}

Smithsonian Libraries

\section{Sponsored by}

Biodiversity Heritage Library

\section{Copyright \& Reuse}

Copyright Status: In Copyright. Digitized with the permission of the rights holder.

Rights Holder: Muséum d'histoire naturelle - Ville de Genève License: http://creativecommons.org/licenses/by-nc-sa/3.0/ Rights: https://www.biodiversitylibrary.org/permissions/

This document was created from content at the Biodiversity Heritage Library, the world's largest open access digital library for biodiversity literature and archives. Visit BHL at https://www.biodiversitylibrary.org. 